\title{
Disagreement and Misunderstanding Across Cultures
}

\author{
HANS ROTT, REGENSBURG
}

\section{Wittgenstein on misunderstanding vs. disagreement}

In the Big Typescript of about 1933, Wittgenstein wrote:

The problem also emerges in this question: How does a misunderstanding become evident? For that's the same as the problem: How does it become evident that I have understood correctly? And that means: How can I explain the meaning? Now the question is: Can a misunderstanding be revealed in one person's affirming what another denies? No, because that's a difference of opinion and it can be adhered to as such. Until we assume that the other person is right ....... What can be removed by an explanation I call a misunderstanding. The explanation of the meaning of a word excludes misunderstandings. ${ }^{1}$

This passage is not particularly transparent. Wittgenstein considers cases of "being at variance" with each other, namely when one person affirms what the other person denies.

What he seems to be saying is that even clear cases of being at variance may be interpreted as harmless cases of "talking past each other", if the first person assumes - as a matter of conversational heuristics - that the other is right. This charitable assumption that what has been said is true

${ }^{1}$ Wittgenstein $(2005,30 \mathrm{e})$. The following text is embedded in the one quoted:

So if to explain the word "lilac" I point to a patch and say "This patch is lilac", can this explanation then work in two ways - on the one hand as a definition that uses the patch as a sign, and on the other as an elucidation? And how is the latter possible? I would have to assume that the other person is telling the truth and seeing the same thing I'm seeing. ...

I could say: If what A told B is the truth, then the word "lilac" must have this meaning. So I can also assume this meaning quasi-hypothetically, and say: if I understand the word in that way, then $\mathrm{A}$ is right. But an ostensive definition corresponds to the "in that way".

We say: "Yes, if the word means that, then the proposition is true". 
may in fact seem to turn "genuine" disagreements into "mere" verbal quibbles.

Similar distinctions like that between Mißverständnis and Meinungsverschiedenheit can be found in two famous papers of the 1970s. In "On the Very Idea of a Conceptual Scheme" (1974), Donald Davidson distinguishes differences in conceptual scheme from differences in belief $f^{2}$, and in "What is a Theory of Meaning?" (1975), Michael Dummett distinguishes disagreements stemming from difference of interpretation from disagreements of substance (disagreements about the facts).

Speakers have a firmly entrenched intuition that there is a fundamental distinction between genuine, substantive disagreement and merely verbal differences (differences in ways of speaking). But what exactly does this distinction between the two consist in? This is the main question of this paper, and I would like to apply it to the problem of intercultural communication. But the question is of course a reasonable one to put for speakers of the same language. We do not expect too varied or too fundamental divergences between speakers of the same language. But let us suppose for the purposes of this paper that only two persons, called "speakers", are involved in a given communication situation. Both disagreement and misunderstanding are spawned by linguistic utterances. If the speakers share the language, one of the speakers affirms $p$, the other one denies it, i.e., affirms the negation $\neg p$. On the face of it, the two persons are at variance with one another. So this appears to be a clear case of disagreement, since after all, speakers of the same language should not assign different meanings to the terms of their common language. But it may happen in the course of a conversation that things are sorted out in such a way that the two persons in the end agree that they have just been using their words in different ways. By assuming that the other one is right ("Until we assume that the other person is right ..."), both partners in conversation can come to agree that the previous disagreement was only a difference of façon de parler (of manner of speaking).

The situation for speakers of different languages or cultures is different. On the one hand, one is prepared to encounter all kinds of discrepancies, disagreements and misunderstandings much more frequently and in much more severe forms than between members of the same culture. After all, different languages mean different cultures, different cultures mean different attitudes, beliefs and desires. So presumably, conflicts are more likely

\footnotetext{
${ }^{2}$ Even though Davidson does not think that there is a clear distinction here.
} 
to occur here than between fellows of the same cultural and linguistic community. This is part of what makes a foreign culture foreign. The situation, however, is more complicated. When two persons are speakers of different languages, they need to make use of a translation that maps the utterances of the one person into the language of the other person. The direct form of disagreement between two speakers of the same language that is codified by a language's negation particle cannot arise. Every sort of apparent conflict of opinion will make itself felt only indirectly, mediated by a translation. And an adequate translation, one might argue, must not only convey what the speaker seems to mean, literally speaking, but also what the speaker really means. Fundamental disagreements seem to be precluded by this method. For could it be that the speaker really has a picture of the world completely different from ours?

The plan of this paper is as follows. First I shall have a brief look at the idea of a good translation and the associated task of ascribing beliefs to a foreign speaker. I shall confront the Kuhnian scepticism about the existence of adequate translations with the Quinean doctrine of the overabundance thereof (Section 2). I shall then look at two rather prominent examples from anthropological research (Section 3). Their analysis favours Quine over Kuhn in that there seem to be common ways of linguistic expression and rationality across vastly different cultures. Then I have a brief look at an artificial example designed to bring out the fact that indeterminacy of translation results in indeterminacy of the distinction between disagreement and misunderstanding (Section 4). The paper ends with some remarks on the difference between translation across foreign cultures and translation within a cultural and linguistic community.

\section{Translation of sentences and ascription of beliefs}

Communication across different cultures requires translation. Our discussion will be restricted to objective assertions, not assertions about ethical or aesthetical matters, for instance. No other speech acts will be considered.

A translation is a function mapping the sentences (or utterances) of one language into the sentences (or utterances) of another language. The central idea is that translation ought to preserve meanings. But preservation of meaning is an idea we will try to make sense of, not an idea to start from. Translations need to be constrained in order to be counted as good or adequate translations. I suggest two important conditions: 
a) Perceptual anchoring. A translation should be anchored in basic perceptual situations. It may be assumed that the same object is seen (heard, felt, smelt, tasted) at a given point of time by different persons in similar ways, and similar perceptions are likely to be grouped under the same or similar concepts. Although the boundaries may be drawn differently by speakers of different languages (sometimes also by speakers of "the same" language), concepts that are closely connected to perceptions should not be wildly at odds with one another. This requirement is motivated by the fact that language learning, whether first or second language, somehow starts with observation sentences.

b) Homomorphy. A translation should be as homomorphic as possible; circuitous, protracted translations are to be avoided. Translation should mimic the syntactical structure of the source sentences in the corresponding sentences of the target language. This, of course, is an ideal that cannot in general be perfectly attained, but still an ideal that is to be pursued. ${ }^{3}$ Homophonic translations go even farther than homomorphic translations: They are identity mappings, and it is plausible (though by no means necessary) to think of the source and target languages as identical. Homophonic translations are very special cases that do not even look like translations.

The two conditions, vague as they are, go a long way toward restricting the range of acceptable translations. How strong are these restrictions? It is very unlikely that they limit the number of acceptable translations of a given corpus of sentences to exactly one. Two other options, both undesirable at first sight, are much more likely. First, the restrictions could be so severe that for all realistic translation tasks, there is not a single translation meeting both Perceptual anchoring and Homomorphy. The pronouncements of speakers of different cultures or languages then are incommensurable, in the sense that there is no common measure to compare their contents, no translation can bridge the gap in Weltanschauung they embody. This calls in question the possibility of successful communication.

\footnotetext{
${ }^{3}$ A perfect correspondence in syntactical structure will only be achieved when objects are similarly categorized by the lexica of the respective languages. For instance, the sentence "this object has colour $C^{\prime \prime}$ in a language having only three colour terms is likely to be translated into a disjunction of sentences in a language with seven colour terms. Thus lexical variance implies syntactical variance. Homomorphic translations are only possible between languages with vocabularies that are in this sense "similar".
} 
Let us call this the Kuhnian option. The other option, in contrast, holds that for all realistic translation tasks, there is an abundance of acceptable translations. Lots of translation schemes satisfy the two constraints, and in fact they all do it, in some sense yet to be explained, equally well. Then we'll have to ask whether it makes any sense to talk of "the right translation" at all or whether there is an essential indeterminacy. This is, of course, the Quinean option.

On the face of it, the Quinean option is much more plausible than the Kuhnian one. The mere fact that we possess zillions of translations of texts - fiction and non-fiction alike -, with which we are more or less content, may serve as an empirical indication that the task of translation is a feasible one. It is true that every translation loses some distinctive characteristics of the original. But this does not discourage us from producing good translations. We rather need to see which further constraints to place on a good translation and whether they can be satisfied simultaneously.

The enterprise of understanding the utterances of a foreigner is not exhausted by the process of translation. Having translated a number of sentences of a speaker, the interpreter needs to make up her mind which beliefs to ascribe to the speaker. This is an interpretational step that is often overlooked. One question relevant here is whether the interpreter should assume that the speaker is logically competent in the sense that he is aware of the implications of what he is saying. Would he realize what his utterances commit him to? Would he be ready to follow them through even if they lead to some strange or even absurd consequences? Or would he rather want to step back if the contents of his utterances are found to be incoherent?

\section{Two anthropological examples}

Genuine disagreement presupposes understanding. What looks like substantive disagreement on the face of it, loses its bite if it turns out that we have just talked past one another. Thus even if we do not know whether a given discrepancy is a case of disagreement or misunderstanding, we need to make plausible anyway that understanding between cultures is possible in principle. I shall now give two anthropological examples that initially seem to favour the Kuhnian option. The cases of Chinese counterfactual reasoning and of Zande logic both became well-known for their apparent 
indication that different people are of so different minds that understanding is hardly ever possible between them.

\subsection{Chinese counterfactuals}

Twenty-five years ago, Alfred Bloom (1981) published a small book with the title The Linguistic Shaping of Thought: A Study in the Impact of Language on Thinking in China and the West. He claimed to have gathered empirical evidence that Chinese people have great difficulties in reasoning from counterfactual assumptions (and thus in performing abstract theoretical reasoning in general), far more than English-speaking people. ${ }^{4}$ As an explanation of this alleged deficiency he offered the fact that the Chinese language does not provide for linguistic markers for counterfactual reasoning. There are some protracted or roundabout ways of expressing counterfactual suppositions, but Chinese does not have anything like the subjunctive conditionals of English. This was supposed to be a paradigmatic instance confirming the notorious Sapir-Whorf hypothesis - a double hypothesis of linguistic relativity and determinism), according to which specific lexical, morphological and syntactic features of a given language determine, to some extent at least, what the speakers are capable of thinking. If Sapir and Whorf were right, speakers subject to the limitations of their language could not think beyond the reach of their language. But then it seems, no translation from a language containing counterfactuals.

Bloom's publication generated a lot of discussion. Counterfactuals were called prime examples of abstract Western reasoning with which Chinese people were alleged to feel very uncomfortable. In the end, however, the inference from the absence of a specific counterfactual conditional construction in the Chinese language to the inability of its speakers to reason counterfactually turned out to be indefensible. Some of Bloom's

${ }^{4}$ Here is one of Bloom's stories and its result: "'Bier was an eighteenth-century European philosopher. There was some contact between the West and China at that time, but very few works of Chinese philosophy had been translated. Bier could not read Chinese, but if he had been able to read Chinese, he would have discovered $B$; what would have most influenced him would have been $C$; once influenced by that Chinese perspective, Bier would then have done $D$,' and so on. The subjects were then asked to check off whether $B, C$, and $D$ actually occurred. The American students gave the correct answer, no, ninety-eight percent of the time; the Chinese students gave the correct answer only seven percent of the time! Bloom concluded that the Chinese language renders its speakers unable to entertain hypothetical false worlds without great mental effort." (Pinker 1995, 56f) 
counterfactually turned out to be indefensible. Some of Bloom's stories were ambiguous, some of his translations into Chinese were not idiomatic, and similar effects could be provoked in Arabic speakers as well as English speakers which both do have a marker for counterfactual conditionals. ${ }^{5}$ The discussion is still going on, but it is fair to say that Bloom's attempt to show that the linguistic peculiarities of a certain language can block its speakers' cognitive powers has failed. If Chinese and Arab speakers sometimes show little inclination to play the games anthropologists would like them to play, this may reflect mainly on the kind of tasks they were put to. ${ }^{6}$

\subsection{Zande reasoning}

So let us take it for granted that other peoples' languages are capable of expressing complex propositions, and let us ask whether the logic on which human reasoning is based is universal. This brings us to another episode of anthropological research that begins with Edward Evans-Pritchard's (1937) famous research on the Azande, a tribe of north central Africa. ${ }^{7}$ This research drew considerable attention from philosophers, as witnessed, for instance, by Peter Winch's (1964) and Charles Taylor's (1982) discussions about the possibility of cross-cultural standards of rationality. Here, however, I want to focus on the discussion following David Bloor's chapter on 'Azande logic and Western Science' in his book Knowledge and Social Imagery (1976). Drawing on Evans-Pritchard, Bloor claimed that the Azande have a logic which is very different from the Western one.

The Azande (as of 1937) believed in witchcraft. More precisely, they believed in a witchcraft-substance in the belly which is inherited from parents to their same-sexed children, i.e., from fathers to sons and from mothers to daughters). While any Zande clan is likely to have some witches (of either sex), no clan is thought to consist only of witches. This, however, seems to run counter to the canons of (our, Western) logic. In the words of Evans-Pritchard (1937, 24):

\footnotetext{
${ }^{5}$ Perhaps the most well-known criticisms of Bloom were provided by Terry Au (1983, 1984), Lisa G. Liu (1985), Yohtaro Takano (1989) and Donna Lardiere (1992).

${ }^{6}$ One of the counterfactual assumptions was "If all circles were large ...". If a disrespect for counterfactuals were typically Chinese, Quine would have had some Chinese traits. For Quine (1960, 222 and 225), the subjunctive conditional, depending on "dramatic projection", has "no place in an austere canonical notation for science".

7 "Zande" is the singular noun and adjectival form of the word and "Azande" is the plural noun. (Jennings 1989, 275)
} 
To our minds it appears evident that if a man is proven a witch the whole of his clan are ipso facto witches, since the Zande clan is a group of persons related biologically to one another through the male line. Azande see the sense of this argument but they do not accept its conclusions, and it would involve the whole notion of witchcraft in a contradiction were they to do so.

While Winch and Taylor addressed the rationality of primitive cultures in a broad perspective, there has been a very careful discussion of precisely this apparent inconsistency in Zande thinking (Triplett 1988, 1994, Jennings 1989, Keita 1993), based on just a few pages in Evans Pritchard (1937, 23-25). This strand of research came to a halt after Triplett's rather convincing diagnosis that "there is no evidence that Zande logical thought processes are different from ours" $(1994,760)$. Triplett's rendering of the Zande reasoning is reconstructed in the Appendix to this paper.

What do these widely discussed anthropological examples show? They provide some (modest) evidence that communication across cultures is not hindered by principal limitations of language and logic. Initial doubts and critical inquiries notwithstanding, it has turned out that counterfactuals can very well be translated "idiomatically" (Au 1983, 1984) into Chinese, and that the Azande do, if pressed, exhibit patterns of reasoning that are quite close to those of Europeans. These examples furnish no empirical evidence for thoughts and patterns of reasoning that cannot be translated from one language into another. So, quite against the initial intentions of Bloom and Bloor, we end up with an argument in favour of the translatability thesis.

Of course there remain deep differences of opinion between the Azande (as of 1937) and us (as of today). But initially the Azande seemed to contradict not only us, but even themselves. This very severe kind of disagreement in the end turned out to be based on a misunderstanding. The reevaluation of the Azande case is not one driven by an "internal" process of reinterpretation. The alleged contradiction disappears after a careful reading of Evans-Pritchard. This happened only with a delay of 50 years, but it did happen. The speculations about a many-valued or paraconsistent logic of the Azande should have been cleared by now (cf. Cooper 1975 vs. Salmon 1978, and da Costa and French 1995). 


\section{An argument for the indeterminacy of translation}

The sentences affirmed by a foreign speaker can be mapped onto sentences of one's own language in essentially different ways. According to Quine, there are multiple non-equivalent translations that are all, in some suitable sense, equally adequate. I shall now offer an explanation why this indeterminacy does not get reduced, even if we introduce a set of additional desiderata:

(i) truth (according to the interpreter)

(ii) consistency (logical coherence 1)

(iii) closure (logical coherence 2)

(iv) informativeness (logical strength)

These desiderata are semantic or logical constraints that play an important role in the task of translation of the foreign speaker's utterances. The numbering here is not supposed to reflect an ordering of importance. On the contrary, I think that various orderings may be reasonable. These four virtues are virtues that the interpreter ascribes to the utterances and beliefs of the speaker, and indirectly they become virtues of the translation. They have to be weighed against each other, and possibly against still more virtues. The desideratum listed first, truth, is the one that reflects whether there is a substantial disagreement (disagreement of belief, disagreement regarding facts) between speaker and interpreter. Whether there is disagreement, or how much disagreement there is, is thus due to how the desideratum of truth compares to the fulfilment of other, competing desiderata. We now turn to an example for illustration.

Suppose we have four utterances which we take as expressing beliefs of a foreign agent. Suppose further for simplicity that we have somehow figured out that the foreigner uses connectives that are identical with our logical connectives not, and and if ... then. The utterances have the following transcriptions:

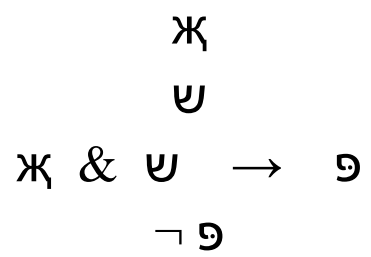

We consider two attempts at translating these sentences. Translation 1 is a completely homomorphic one. It translates (putatively) atomic sentences into atomic sentences (column 1a). What the interpreter really has to pro- 
vide here, however, is not just a translation, i.e., a mapping taking the four sentences of the foreign language and yielding four corresponding sentences of the interpreter's own language. Ultimately, in understanding the other, the interpreter rather needs to make sense of what is said, and thus he needs to ascribe beliefs to the foreign speaker. Aiming at desideratum (iii), we will articulate the speaker's commitments by including all logical consequences of what she has said (column 1b). Discovering that the sentences taken literally are inconsistent, we perhaps want to step back and say that the speaker really only believes three out of the four sentences. Since we cannot say which, we go for the disjunction (column 1c).

\begin{tabular}{|c|c|c|}
\hline $1 \mathrm{a}$ & $1 \mathrm{~b}$ (closure) & 1c (cautious disjunction) \\
\hline$p$ & & $\mathrm{Cn}((p \& q \& r) \vee$ \\
$q$ & $(p \& q \& \neg r) \vee$ \\
$p \& q \rightarrow r$ & $\mathrm{Cn}(\perp) \begin{array}{c}\text { inconsistent } \\
\text { theory }\end{array}$ & $(p \& \neg q \& \neg r) \vee$ \\
& & $(\neg p \& q \& \neg r))$ \\
& & $\mathrm{Cn}((p \vee q) \&(r \rightarrow p \& q))$ \\
\hline
\end{tabular}

The example gets more vivid if we assign some content to the letters. Let $p$ stand for "The land is suffering from a drought", $q$ for "Rain will come soon" and $r$ for "The harvest will be good". Now add $s$ standing for "The gods are angry with us". Assume that the alternative translation 2 is not fully homomorphic, but based on the methodological hypothesis that some categorical statements may really be hedged by an implicit precondition. Indeed they have to be understood as qualified by the clause "if the gods are not angry with us" $(\neg s)$. Suppose that in our case $q$ is for some reason a good candidate for hedging, because we have come to think that all predictions concerning the weather are to be qualified in this way. So we translate the atomic sentence $ש$ by $\neg s \rightarrow q$ (column $2 \mathrm{a}$ ). Then we again ascribe to the speaker all consequences to the of the translated sentences (column 2b) which are consistent, so there is no need for a third column.

\begin{tabular}{|c|c|c|}
\hline $2 \mathrm{a}$ & $2 \mathrm{~b}$ (closure) & \\
\hline$p$ & & \\
$\neg s \rightarrow q$ & $\mathrm{Cn}(p, \neg q, \neg r, s)$ & \\
$p \& q \rightarrow r$ & & \\
$\neg r$ & & \\
\hline
\end{tabular}


Now let us compare the virtues of these translations. Suppose that from the interpreter's point of view, i.e., according to the interpreter's beliefs, the following propositions are true: $p, q, r$ and $\neg s$. As long as we deal with a set of sentences that is not logically closed (i.e., no "theory" in the logician's sense), it makes sense to count discrepancies between foreign speaker and interpreter. If all sentences but one are true by the interpreter's lights, we will say that the criterion (i) of truth is reasonably well satisfied (and write ' \pm ' for short). Here is a table of the semantic virtues of our respective translations:

\begin{tabular}{|c|c|c|c|c|}
\hline translation & truth & consistency & closure & strength \\
\hline $1 \mathrm{a}$ & \pm & - & - & + \\
\hline $1 \mathrm{~b}$ & - & - & + & + \\
\hline $1 \mathrm{c}$ & + & + & + & - \\
\hline $2 \mathrm{a}$ & \pm & + & - & + \\
\hline $2 \mathrm{~b}$ & - & + & + & + \\
\hline
\end{tabular}

While belief ascription $2 \mathrm{~b}$ has some advantages over ascriptions based on the homomorphic translation, it results in a lot of disagreement between speaker and interpreter. On the whole, the second translation may look slightly better than the first. But remember that there is a price to be paid for this: We had to compromise the syntactic requirement of homomorphic translation. Different translation strategies give markedly different results, and it is by no means clear which desiderata are the most important ones. So we have got an idea how the problem of translation admits of multiple solutions due to several partially conflicting desiderata. The indeterminacy of translation gives rise to a corresponding indeterminacy concerning the substantive disagreements between speaker and interpreter.

\section{Conclusion}

Contacts with foreign cultures provide us with valuable new experiences that widen our perspectives. By the same token, interacting with foreign cultures may be disconcerting. We may not understand what other people are saying and doing. Supposing even that we master the foreign language or have an interpreter to help us, we often ask ourselves: What do they mean by their words and sentences, what do they believe and want? The challenge is to make sense of other cultures as a whole. 
Problems of verbal communication may manifest themselves in "discrepancies", that is, in disagreements or misunderstandings. Communication problems impeding dialogues may lead to all sorts of conflicts. Thus, when communication problems arise, it appears crucial to separate "genuine" disagreement from "mere" misunderstanding. I have focussed in this paper on the question when we can be said to disagree with some representative of a foreign culture. ${ }^{8}$ When do two persons of different cultures "really" disagree?

Disagreement presupposes (some very basic sort of) understanding. In order to identify a disagreement between speakers of different languages, some sort of translation between them is needed. I have argued that there is anthropological evidence that communication across different cultures and languages is possible, since (i) the degrees of sophistication in thinking or talking are not fundamentally different between different cultures (the Chinese counterfactual case) and (ii) the basic logics used are not fundamentally different (the Zande logic case). Disagreements and misunderstandings are not clearly separable, because (iii) it is only relative to a given translational scheme that one can talk of a member of one culture denying what a member of another culture affirms (the made-up story).

The point of my paper comes out clearly if we consider communication across languages rather than communication in a single language. However, it transfers to speakers of the same language (or, at least, of what looks like the same language). Radical translation, as Quine used to say, begins at home.

To this we add that the banal observation that disagreement begins at home. Intuitively, we expect to come across many disagreements with foreigners. But in a sense, it is harder for members of different cultures to disagree than for members of the same culture. Translations are there to solve communication problems, and as such they have the potential to iron out, or at least to cushion, differences of opinion. Within a language, there are well-established markers, negation words like "not" in particular, serving as devices for the direct expression of disagreements. They are not likely to be translated away. The problem of how to tell apart disagreements from misunderstandings is hidden by our fixation on homophonic translation of

\footnotetext{
${ }^{8}$ I have said nothing about moral or aesthetic matters where we are likely to acknowledge legitimate disagreements even within our own culture. In aesthetic matters, we tolerate and even tend to welcome disagreements.
} 
people sharing our culture. ${ }^{9}$ But translation across subcultures may in fact be just as hard as translation across cultures.

Agreeing to disagree is one of the hardest exercises we know, and if it succeeds, it is an agreement about no matter of fact.

\section{REFERENCES}

Au, T. 1983 "Chinese and English counterfactuals: The Sapir-Whorf hypothesis revisited", Cognition, 15, 155-187.

- 1984 "Counterfactuals: In reply to Alfred Bloom", Cognition, 17, 289-302.

Bloom, A.H. 1981 The Linguistic Shaping of Thought: A Study in the Impact of Language on Thinking in China and the West, Hillsdale, NJ: Erlbaum.

Bloor, D. 1976 Knowledge and Social Imagery, London: Routledge and Kegan Paul.

da Costa, N. and French, S. 1995 "Partial Structures and the Logic of Azande", American Philosophical Quarterly, 32, 325-339.

Cooper, D.E. 1975, "Alternative Logic in 'Primitive Thought'", Man (N.S.), 10, 238256.

Davidson, D. 1974, "On the Very Idea of a Conceptual Scheme", Proceedings and Addresses of the American Philosophical Association, 47, 5-20.

Dummett, M. 1975, "What is a Theory of Meaning?", in S. Guttenplan (ed.), Mind and Language, Oxford: Clarendon Press, 97-138.

Evans-Pritchard, E.E. 1937 Witchcraft, Oracles and Magic Among the Azande, Oxford: Clarendon Press.

Jennings, R.C. 1989 "Zande Logic and Western Logic", British Journal for the Philosophy of Science, 40, 275-285.

Keita, L. 1993 "Jennings and Zande Logic: A Note", British Journal for the Philosophy of Science, 40, 275-285.

Lardiere, D. 1992 "On the linguistic shaping of thought: Another response to Alfred Bloom", Language in Society, 21, 231-251.

Liu, L.G. 1985 "Reasoning counterfactually in Chinese: Are there any obstacles?", Cognition, 21, 239-270.

Pinker, S. 2000 The Language Instinct, First Perennial Classics Edition, New York: Harper Collins.

\footnotetext{
${ }^{9}$ This is not to deny that in particularly striking cases, we do give up homophonic translations. Cf. Quine $(1960,59)$ : "The maxim of translation underlying all this is that assertions startlingly false on the face of them are likely to turn on hidden differences of language. This maxim is strong enough in all of us to swerve us even from the homophonic method that is so fundamental to the very acquisition and use of one's mother tongue. The common sense behind the maxim is that one's interlocutor's silliness, beyond a certain point, is less likely than bad translation-or, in the domestic case, linguistic divergence."
} 
Quine, W.V.O. 1960 Word and Object, Cambridge, Mass.: MIT Press.

Salmon, M.H. 1978, "Do Azande and Nuer Use Non-Standard Logic?", Man (N.S.), $13,444-454$.

Takano, Y. 1989 "Methodological problems in cross-cultural studies of linguistic relativity", Cognition, 31, 141-162.

Taylor, C. 1982 "Rationality", in M. Hollis and S. Lukes (eds.), Rationality and Relativism, Cambridge, Mass.: MIT Press, 87-105.

Triplett, T. 1988 "Azande Logic versus Western Logic?", British Journal for the Philosophy of Science, 44, 361-366.

- 1994 "Is There Anthropological Evidence that Logic Is Culturally Relative? Remarks on Bloor, Jennings, and Evans-Pritchard", British Journal for the Philosophy of Science, 45, 749-760.

Winch, P. 1964 "Understanding a Primitive Society", American Philosophical Quarterly, 1, 307-324.

Wittgenstein, L. 2005 The Big Typescript: TS 213, G. Luckhardt and M. Aue (eds.), German-English Edition, Oxford: Blackwell.

\section{Appendix: A logical reconstruction of the Zande case}

For the formalization I use as predicates the capitalized first letters of 'Witch', 'Male', 'Female', 'Parent' and 'member of $C$ lan $C$ '; 'S' stands for 'possesses witchcraftsubstance', ' $P *$ ' for the transitive closure of $P$ (to be read as 'ancestor'). We first formalize Triplett's $(1988,364)$ reconstruction of a central Zande argument as reported by Evans-Pritchard (1937, 24):

\section{The argument}

(1') Every witch has the witchcraft-substance, and everyone possessing the witchcraft-substance is a witch.

(2) The witchcraft-substance is always inherited by the same-sexed children of a witch.

(3) Male $A$ and female $B$ were both witches.

(4) Everyone in clan $C$ descends from $A$ or $B$.

(5) Everyone in clan $C$ is a witch.
Formalization

$\forall x(W x \rightarrow S x) \&$

$\forall x(S x \rightarrow W x)$

$\forall x \forall y(P x y \&((M x \& M y) \vee$ $(F x \& F y)) \rightarrow(S x \rightarrow S y))$

$(M a \& W a) \&(F b \& W b)$

$\forall x\left(C x \rightarrow P^{*} a x \& P^{*} b x\right)$
$\forall x(C x \rightarrow W x)$

It is an easy exercise to check that this argument is logically valid. Prima facie, the Azande seem to accept premises (1') - (4) but refuse to draw the conclusion, apparently in order to remain consistent with their belief that not everyone in the clan is a witch. In particular, the persons interviewed want to avoid the conclusion that they are witches. In this sense, it seems that they do not see the contradiction in their beliefs. However, as Triplett points out, consistency is saved if we replace premise (1') by the weaker premise 
(1) Every witch has the witchcraft-substance. $\quad \forall x(W x \rightarrow S x)$

(1) - (4) do not imply (5). (1) is actually in accordance with the "cool witchcraftsubstance doctrine" (Triplett 1994, 756) reported by Evans-Pritchard (1937, 25). So Triplett saves Zande reasoning, but I don't think he is hermeneutically careful enough. Here is an improved reconstruction that is somewhat more faithful to Evans-Pritchard's original account:

\section{The argument improved}

$\left(1^{*}\right)$ Every witch has the witchcraft-substance.

(2*) The witchcraft-substance is always inherited by the same-sexed children of a witch, and this is the only way of getting witchcraft-substance.

$\left(3^{*}\right)$ There is a male and a female witch in the clan.

$\left(4^{*}\right) \quad$ Any two members in clan $C$ are related by common male and female ancestors.

$\left(5^{*}\right)$ Everyone in clan $C$ is a witch.

$\left(1^{*}\right) \quad \forall x(W x \rightarrow S x)$

(2*) $\quad \forall x \forall y(P x y \&((M x \& M y) \vee(F x \& F y)) \rightarrow(S x \leftrightarrow S y))$

(3*) $\exists x(C x \& M x \& W x) \& \exists y(C y \& F y \& W y)$

(4*) $\forall x \forall y\left(C x \& C y \rightarrow \exists z \exists z^{\prime}\left(M z \& W z^{\prime} \& P^{*} z x \& P^{*} z y \& P^{*} z^{\prime} x \& P^{*} z^{\prime} y\right)\right)$

$\left(5^{*}\right) \quad \forall x(C x \rightarrow W x)$

The biconditional in $\left(2^{*}\right)$ strengthens (2) by taking care of the fact that the Azande say that "witchcraft does not trouble a person born free from it by entering into him" (Evans-Pritchard 1937, p. 23). I interpret this as meaning that the witchcraft-substance can only be had by inheriting it from one's same-sexed parent. $\left(3^{*}\right)$ and $\left(4^{*}\right)$ avoid the direct assumption expressed by (3) and (4) that each clan is formed of the offspring of a certain pair of witches. $\left(3^{*}\right)$ says that there are both male and female witches in the clan, and $\left(4^{*}\right)$ says that any two members of the clan have common male and female ancestors. (3*) and $\left(4^{*}\right)$ are weaker than (3) and (4), respectively. As with Triplett's reconstruction in terms of (1) - (4), the argument is invalid as it stands. It would be valid if $\left(1^{*}\right)$ were strengthened to $\left(1^{\prime}\right)$ which is denied by the Azande. The only thing that can be derived is that all members of the clan possess the witchcraft-substance. That substance may, however, be "cool" (i.e., inactive) in most individuals.

There are other strategies to ward off the charge of inconsistency (e.g., by suspecting a male witch's mother of adultery). In any case, the imputation that the Azande have a different logic from ours is ill-founded. Their reasoning may be ad hoc, but it does not violate the canons of classical logic. 
